\title{
ROBUST MULTIBASELINE INSAR OPTIMIZATION
}

\author{
Yuanyuan Wang $^{1}$, Xiao Xiang Zhu ${ }^{1,2}$ \\ 1. Signal Processing in Earth Observation, Technical University of Munich, Germany \\ 2. Remote Sensing Technology Institute, German Aerospace Center, Germany
}

\begin{abstract}
Multibaseline SAR interferometry may face unmodeled interferometric phase such as unmodeled motion phase and uncompensated atmospheric phase, as well as non-Gaussian statistics in the context of distributed scatterer. We developed the robust InSAR optimization (RIO) [1] framework to systematically tackle these issues. Experiments show that RIO outperforms the current multibaseline InSAR methods in terms of the variance of the phase history parameters estimates for contaminated observations, while still keeping a relative efficiency of $80 \%$ for outlier-free observations.
\end{abstract}

Index Terms - robust estimation, M-estimator, RIO, covariance matrix, RME, InSAR, SAR

\section{INTRODUCTION}

Multibaseline synthetic aperture radar interferometry (InSAR) techniques are widely employed for millimeter-level long-term deformation monitoring and 3-D reconstruction of large areas. For example, persistent scatterer interferometry (PSI) [2], [3], and SqueeSAR [4], [5], which utilizes persistent scatterers (PS) and distributed scatterers (DS), respectively. In general, millimeter accuracy of the yearly linear deformation rate can be achieved with respect to a reference point [2], [6], [7].

However, these accuracies refer to the optimal estimators derived based on the following assumptions:

- Correct interferometric phase history model. This is sometimes not fulfilled, e.g. unmodeled motion phase. Unmodeled interferometric phase leads to biased parameters estimates.

- Gaussian-distributed data. For instance, PS is modeled as a deterministic signal with additive zero-mean white complex circular Gaussian (CCG) noise [2], while DS is modeled as correlated zero-mean CCG [4], [8]. NonGaussian scatterers act as outliers under such assumption.

Violation of these assumptions greatly compromises the performance of the estimators. RIO solves the problem by introducing the following two points:
- It replaces the maximum likelihood estimator (MLE) for Gaussian distribution which minimizes the sum of the squared residuals with an M-estimator [9] which minimizes the sum of a customized function $\rho(x)$ of the residuals.

- Should DS be exploited, the sample covariance matrix $\hat{\mathbf{C}}$ is replaced with the robust rank M-estimator (RME) of the covariance: $\hat{\mathbf{C}}_{R M E}$ proposed in this paper. $\hat{\mathbf{C}}_{R M E}$ is robust against both outlier and samples with non-stationary phase.

\section{ROBUST INSAR OPTIMIZATION}

\subsection{Robust Estimators for Phase History Parameters}

\subsubsection{Persistence Scatterer}

The periodogram is one of the most common estimators for PS phase history parameters $\boldsymbol{\theta}$ :

$$
\hat{\boldsymbol{\theta}}=\arg \max _{\boldsymbol{\theta}}\left\{\left|N^{-1} \sum_{n=1}^{N} g_{n} \exp \left(-j \varphi_{n}(\boldsymbol{\theta})\right)\right|\right\}
$$

Where $N$ is the number of images, $g_{n}$ and $\varphi_{n}$ are the complex pixel value and the modeled phase of the $n$th image, respectively. Equation (2.1) is actually the MLE under the assumption of additive white CCG noise [10].

In case of insufficient order of phase history model, the unmodeled phase renders the noise of PS no longer Gaussian. Therefore, a robust estimator is required in such case. RIO introduces the following estimator to deal with possible large phase error:

$$
\hat{\boldsymbol{\theta}}=\arg \min _{\boldsymbol{\theta}} \sum_{i=1}^{N} \rho\left(\frac{\operatorname{Re}\left[\varepsilon_{i}(\boldsymbol{\theta})\right]}{\sigma_{R}}\right)+\rho\left(\frac{\operatorname{Im}\left[\varepsilon_{i}(\boldsymbol{\theta})\right]}{\sigma_{I}}\right)
$$

where $\rho(\bullet)$ is a robust loss function, e.g. Huber's loss function or Tukey's biweight loss, the residual $\varepsilon_{i}(\boldsymbol{\theta})$ is $g_{i}-\exp \left(-j \varphi_{i}(\boldsymbol{\theta})\right), \operatorname{Re}[\cdot], \operatorname{Im}[\cdot]$ are the real and imaginary parts, and $\sigma_{R}$ and $\sigma_{I}$ are the corresponding standard deviations of the real and imaginary parts. Equation (2.2) is 
solved iteratively, where $\sigma_{R}$ and $\sigma_{I}$ are updated at each iteration.

\subsubsection{Distributed Scatterer}

The MLE for DS phase history parameters is as follows:

$$
\hat{\boldsymbol{\theta}}=\arg \min _{\boldsymbol{\theta}}\left\{\mathbf{g}^{H} \boldsymbol{\Phi}(\boldsymbol{\theta})|\hat{\mathbf{C}}|^{-1} \boldsymbol{\Phi}(\boldsymbol{\theta})^{H} \mathbf{g}\right\}
$$

where $\boldsymbol{\Phi}(\boldsymbol{\theta})$ is the diagonal matrix containing the modelled phase of $\mathbf{g},|\cdot|$ is the element-wise absolute value operator, and $\hat{\mathbf{C}}$ is the estimated covariance matrix.

RIO introduces a robust diagonal weighting matrix $\mathbf{W}$ into the MLE:

$$
\hat{\boldsymbol{\theta}}=\arg \min _{\boldsymbol{\theta}}\left\{\boldsymbol{\varepsilon}^{H}(\boldsymbol{\theta}) \mathbf{W} \boldsymbol{\varepsilon}(\boldsymbol{\theta})\right\}
$$

where the residual is the whitened DS observations, i.e.:

$$
\boldsymbol{\varepsilon}(\boldsymbol{\theta})=|\hat{\mathbf{C}}|^{-1 / 2} \boldsymbol{\Phi}(\boldsymbol{\theta})^{H} \mathbf{g} .
$$

The residual is better to be whitened with a robust covariance matrix estimate, such as the $\hat{\mathbf{C}}_{R M E}$ which will be covered in Section 2.2.

W downweights the observations with large unmodeled phase. Given a robust loss function $\rho(x)$, each element of $\mathbf{W}$ is can be derived from the corresponding element of the residual $\boldsymbol{\varepsilon}(\boldsymbol{\theta})$ as follows [1]:

$$
W_{i}=\rho^{\prime}\left(\varepsilon_{i}(\boldsymbol{\theta})\right) / \varepsilon_{i}(\boldsymbol{\theta})
$$

where $\rho^{\prime}(x)$ is the first order derivative of $\rho(x)$.

However, the weights calculated based on a single DS pixel are not optimal. It should be calculated based on the expected residuals $\overline{\boldsymbol{\varepsilon}}$ of the whole DS neighbourhood. The expected residuals are better to be robustly estimated using equation (2.7), due to the possible outliers in the neighbourhood:

$$
\overline{\boldsymbol{\varepsilon}}=\left(\sum_{m=1}^{M} w^{m}\right)^{-1} \sum_{m=1}^{M} w^{m} \boldsymbol{\varepsilon}\left(\hat{\boldsymbol{\theta}}^{m}\right)
$$

where the superscript $m$ denotes the sample number in the neighbourhood, and $w^{m}$ is a robust weight. The complete final estimator should be as follows:

$$
\hat{\boldsymbol{\theta}}^{m}=\arg \min _{\boldsymbol{\theta}^{m}}\left\{\boldsymbol{\varepsilon}^{H}\left(\boldsymbol{\theta}^{m}\right) \mathbf{W}(\overline{\boldsymbol{\varepsilon}}) \boldsymbol{\varepsilon}\left(\boldsymbol{\theta}^{m}\right)\right\} .
$$

Similar to (2.2), equation (2.8) is also solved iteratively. The weighting matrix is updated at each iteration. Its computation should start with a selected DS neighbourhood which jointly determines a single weighting matrix. This matrix is used for the parameters retrieval of each single-look DS observation vector in the neighbourhood. The weighting matrix is then updated according to all the estimates in the neighbourhood.

\subsection{Robust Covariance Estimators}

In a similar vein, the covariance estimation of non-Gaussian scatterers can also be robustified using an M-estimator.

The M-estimator of a covariance matrix is basically an iteratively reweighted sample covariance matrix [11]:

$$
\hat{\mathbf{C}}_{k+1}=\frac{1}{M} \sum_{m=1}^{M} w\left(\varepsilon_{m}^{2}\left(\hat{\mathbf{C}}_{k}\right)\right) \mathbf{g}_{m} \mathbf{g}_{m}^{H}
$$

where $k$ is the iteration index, and $w(x)$ is a weighting function. $w(x)$ downweights highly deviating samples whose whitened version $\varepsilon_{m}\left(\hat{\mathbf{C}}_{k}\right)=\left\|\hat{\mathbf{C}}_{k}^{-1 / 2} \mathbf{g}_{m}\right\|$ is large in magnitude, which greatly depends on the intensities of $\mathbf{g}_{m}$.

For samples with non-stationary interferometric phase caused by topography, deformation, etc., RIO defines a new quantity: complex rank of InSAR multivariate as follows [1]:

$$
\hat{\mathbf{r}}_{m}=\frac{1}{J} \sum_{j=1}^{J} \mathbf{g}_{m} \cdot \mathbf{g}_{j}^{*} /\left\|\mathbf{g}_{m} \cdot \mathbf{g}_{j}^{*}\right\|
$$

where $\mathbf{g}_{j}$ is the direct neighbourhood of $\mathbf{g}_{m}$, and the • denotes the element-wise product. Through the multiplication of the sample with the complex conjugate of its direct neighbour, the deterministic phase is mitigated. Based on (2.10), we can define the RME of the covariance matrix analogous to (2.9) as follows [1]:

$$
\hat{\mathbf{C}}_{R M E}=\frac{1}{M} \sum_{m=1}^{M} w\left(\varepsilon_{m}^{2}\right) \hat{\mathbf{r}}_{m} \hat{\mathbf{r}}_{m}^{H}
$$

where the iteration index $k$ is dropped for simplicity. RME is a fourth-order descriptor of the sample statistics. It can be proven that the element wise square root of $\left|\hat{\mathbf{C}}_{R M E}\right|$ approaches $\left|\hat{\mathbf{C}}_{M L E}\right|$ asymptotically under CCG for using one direct neighbourhood [1]. Therefore, element-wise square root on $\left|\hat{\mathbf{C}}_{R M E}\right|$ should be taken after equation (2.11).

\section{PRACTICAL DEMONSTRATION}

\subsection{Robustness Against Unmodeled Phase}

An area in Las Vegas with significant non-linear motion was selected as the test area. Its amplitude image is shown in Figure 1(a). As an example, the deformation phase history of the pixel marked by the yellow cross w.r.t. a 
reference point nearby is shown in Figure 1(b). As we can see, not only the magnitude of the motion is large, but also the motion is very complex. If only linear motion model is considered, the unmodeled motion phase is equivalent to large phase error. Non-robust estimators, i.e. MLE, will give biased estimates.

The upper subfigures of Figure 2 compare the result of RIO and the ordinary MLE when only the linear motion model is considered. The robust estimates correctly reconstruct the subsiding bowl, whereas the MLE estimates are heavily biased by the unmodeled motion of the building. The advantage of the robust estimator is clearer in the lower subfigures of Figure 2 which is the bias of the estimates w.r.t. the reference linear deformation rate estimated using a multi-component nonlinear motion model.

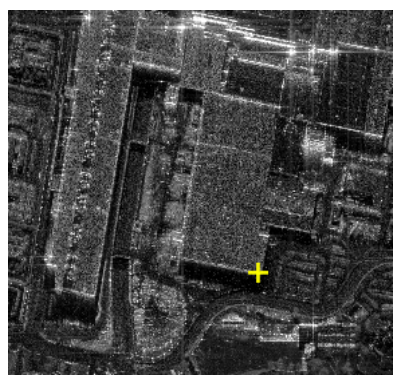

(a)

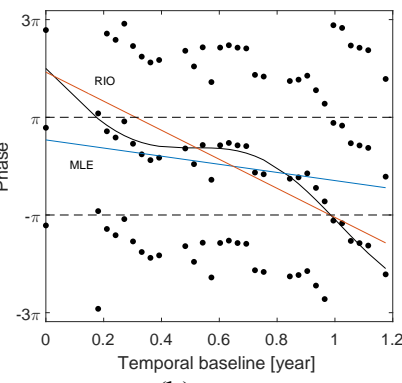

(b)
Figure 1. (a) the amplitude image of the test area with significant non-linear motion, and (b) the deformation phase history of the pixel marked by yellow cross in (a). The black dots are the wrapped deformation phases and its duplicates by adding and subtracting $2 \pi$. The black curve is a non-linear fit to the phase history, and the red and blue lines are linear fittings using RIO and MLE, respectively.
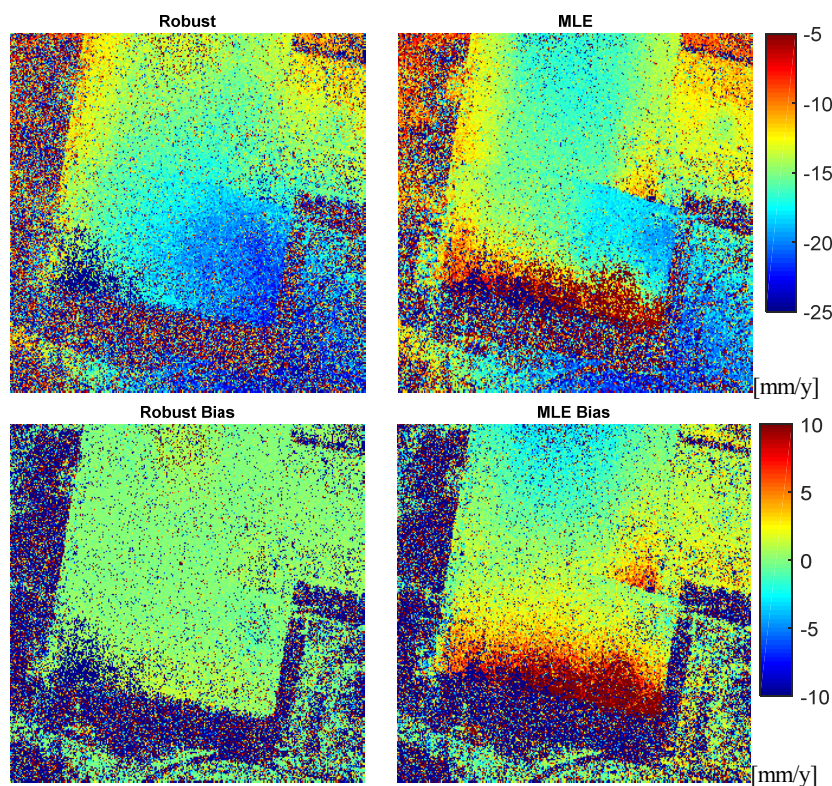

Figure 2. Upper: linear deformation rate estimates by robust estimator and ordinary MLE, and lower: the bias of the robust and non-robust estimates to the "ground truth" (linear deformation rate estimated considering the non-linear motion model). Unit: [mm/year].

\subsection{Robustness against Non-Gaussian Scatterers}

A test area in the volcanic region Campi Flegrei was selected for this test. The test area consists of a single road in the middle which usually appears as DS in X-band SAR images. Figure 3 shows the linear deformation rate of the DSs estimated using the classical sample covariance matrix $\hat{\mathbf{C}}_{M L E}$ (left column) and that using the proposed $\hat{\mathbf{C}}_{R M E}$ (right column). Identical samples were used for estimating these two covariance matrices. They were adaptively selected with the KolmogorovSmirnov (KS) test using ten amplitude images. Due to the low detection rate caused by the small number of images, we expect a non-negligible number of outliers in the selected samples. The same ordinary DS MLE was employed to estimate the linear deformation rate in both cases. Therefore, any improvement was solely credited to the use of a more robust covariance matrix estimate.

The improvement is clearly demonstrated. Homogeneous deformation rates are expected, as the size of the test area is roughly one hundred meter. However, many estimates appear in subfigure (a) as salt-and-pepper noise. We believe this is due to the low detection rate of the KS test, and the non-stationarity of the samples. In contrast, homogenous deformation rate of the road is shown in subfigure (b).

For further comparison, the histograms of the linear deformation rates of the road were plotted in Figure 3(c) and (d) respectively. When using $\hat{\mathbf{C}}_{M L E}$, many local peaks appear in the histogram which should not correspond to the deformation signal. With $\hat{\mathbf{C}}_{R M E}$, results are considerably more homogenous, and thus more reasonable.

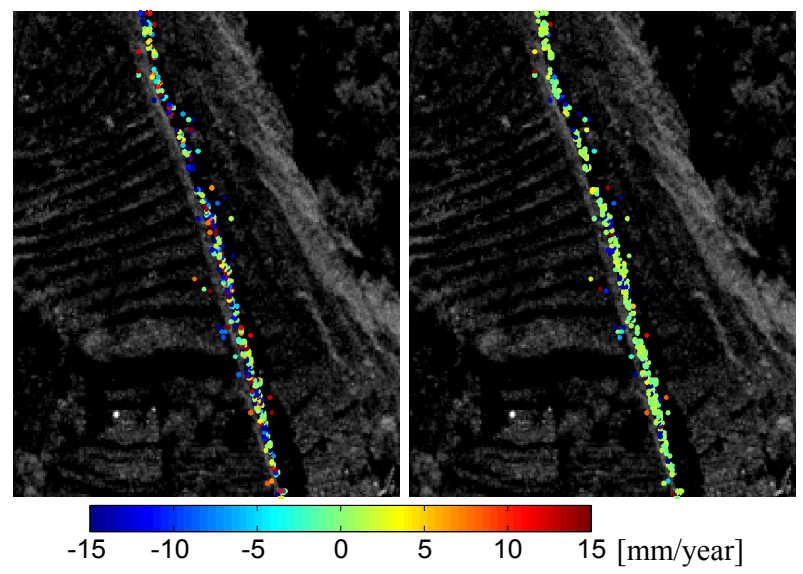

(a)

(b) 


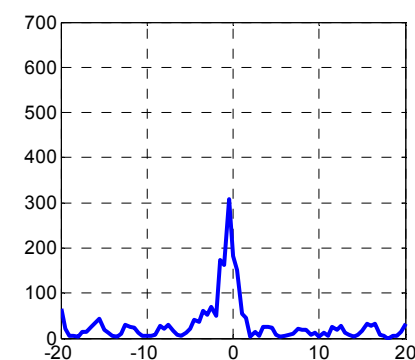

(c)

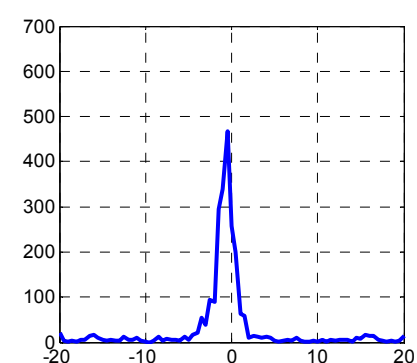

(d)
Figure 3. (a) and (b) comparison of the linear deformation rate of the two test sites estimated using the ordinary $\hat{\mathrm{C}}_{M L E}$ and the proposed $\hat{\mathbf{C}}_{R M E}$, respectively, and (c) and (d) the corresponding histograms of the linear deformation rates in Figure 3 (a) (b).

\subsection{Large Area Processing}

RIO has been implemented for parallel processing on large servers. Figure 4 is the result of the entire area of the volcanic region Campi Flegrei. The stack contains 34 TS-X high resolution spotlight images, spanning from Dec. 2009 to Mar. 2012. The result of using only the PS is shown as the upper plot of Figure 4. The lower one is the result using the proposed RME on DS, combined with the PS result. It retrieves 15 times more scatterers than using the PS only. For good visualization, only $10 \%$ of the points from either method are plotted in Figure 4, and the point size is kept the same for both subplots.
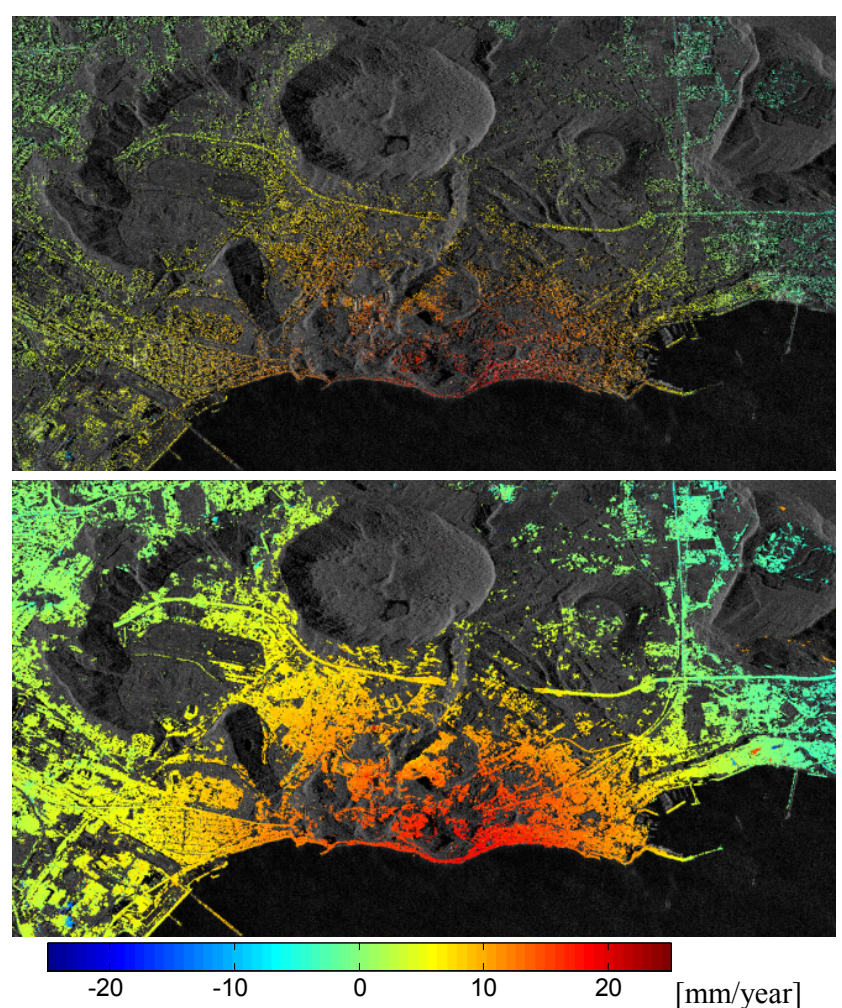

Figure 4. Linear deformation rate of the super volcano Campi Flegrei using only the PS (upper), and using PS+DS with the proposed RME covariance matrix estimation method (lower).

\section{CONCLUSION AND OUTLOOK}

RIO is designed for the robust retrieval of the phase history parameters, e.g. deformation parameters, from multibaseline SAR image stacks. It can be easily applied to most of the current multibaseline InSAR techniques, such PSI, SqueeSAR, and TomoSAR. RIO outperforms these methods by 7 to 35 times [1] in terms of the estimates accuracy for outlier-heavy observations. But it is also able to maintain a relative efficiency of $80 \%$ for outlier-free observations. Therefore, RIO is perfectly suited for large data processing.

We are currently developing a classification algorithm to detect outlier pixels so that only these pixels will be processed by RIO.

\section{REFERENCES}

[1] Y. Wang and X. X. Zhu, "Robust Estimators for Multipass SAR Interferometry," IEEE Trans. Geosci. Remote Sens., vol. 54, no. 2, 2015.

[2] A. Ferretti, C. Prati, and F. Rocca, "Permanent scatterers in SAR interferometry," IEEE Trans. Geosci. Remote Sens., vol. 39, no. 1, pp. 8-20, Jan. 2001.

[3] N. Adam, B. Kampes, M. Eineder, J. Worawattanamateekul, and M. Kircher, "The development of a scientific permanent scatterer system," in ISPRS Workshop High Resolution Mapping from Space, Hannover, Germany, 2003, vol. 2003, p. 6.

[4] A. Ferretti, A. Fumagalli, F. Novali, C. Prati, F. Rocca, and A. Rucci, "A New Algorithm for Processing Interferometric Data-Stacks: SqueeSAR," IEEE Trans. Geosci. Remote Sens., vol. 49, no. 9, pp. 3460-3470, Sep. 2011.

[5] Y. Wang, X. Zhu, and R. Bamler, "Retrieval of Phase History Parameters from Distributed Scatterers in Urban Areas Using Very High Resolution SAR Data," ISPRS J. Photogramm. Remote Sens., vol. 73, pp. 89-99, Sep. 2012.

[6] R. Bamler, M. Eineder, N. Adam, X. Zhu, and S. Gernhardt, "Interferometric Potential of High Resolution Spaceborne SAR," Photogramm. - Fernerkund. - Geoinformation, vol. 2009, no. 5, pp. 407-419, Nov. 2009.

[7] X. Zhu and R. Bamler, "Very High Resolution Spaceborne SAR Tomography in Urban Environment," IEEE Trans. Geosci. Remote Sens., vol. 48, no. 12, pp. 4296-4308, 2010.

[8] R. Bamler and P. Hartl, "Synthetic aperture radar interferometry," Inverse Probl., vol. 14, no. 4, p. R1, 1998.

[9] P. J. Huber, Robust Statistics. John Wiley \& Sons, 1981.

[10] D. Rife and R. R. Boorstyn, "Single tone parameter estimation from discrete-time observations," Inf. Theory IEEE Trans. On, vol. 20, no. 5, pp. 591-598, 1974.

[11] E. Ollila and V. Koivunen, "Influence functions for array covariance matrix estimators," in Statistical Signal Processing, 2003 IEEE Workshop on, 2003, pp. 462-465. 